\title{
Crystal Structure of the $\mathrm{N}$-Acetyltransferase Domain of Human $\mathrm{N}$-Acetyl-L-Glutamate Synthase in Complex with $\mathrm{N}$-Acetyl-L-Glutamate Provides Insights into Its Catalytic and Regulatory Mechanisms
}

\author{
Gengxiang Zhao', Zhongmin Jin $^{2}$, Norma M. Allewell ${ }^{3}$, Mendel Tuchman ${ }^{1}$, Dashuang Shi ${ }^{1 *}$ \\ 1 Center for Genetic Medicine Research and Department of Integrative Systems Biology, Children's National Medical Center, The George Washington University, \\ Washington, D. C., United States of America, 2 Southeast Regional Collaborative Access Team, Advanced Photon Source, Argonne National Laboratory, Argonne, Illinois, \\ United States of America, 3 Department of Cell Biology and Molecular Genetics and Department of Chemistry and Biochemistry, College of Computer, Mathematical, and \\ Natural Sciences, University of Maryland, College Park, Maryland, United States of America
}

\begin{abstract}
$\mathrm{N}$-acetylglutamate synthase (NAGS) catalyzes the conversion of AcCoA and L-glutamate to CoA and $\mathrm{N}$-acetyl-L-glutamate (NAG), an obligate cofactor for carbamyl phosphate synthetase I (CPSI) in the urea cycle. NAGS deficiency results in elevated levels of plasma ammonia which is neurotoxic. We report herein the first crystal structure of human NAGS, that of the catalytic $N$-acetyltransferase (hNAT) domain with $N$-acetyl-L-glutamate bound at $2.1 \AA$ resolution. Functional studies indicate that the hNAT domain retains catalytic activity in the absence of the amino acid kinase (AAK) domain. Instead, the major functions of the AAK domain appear to be providing a binding site for the allosteric activator, L-arginine, and an $N$ terminal proline-rich motif that is likely to function in signal transduction to CPS1. Crystalline hNAT forms a dimer similar to the NAT-NAT dimers that form in crystals of bifunctional $N$-acetylglutamate synthase/kinase (NAGS/K) from Maricaulis maris and also exists as a dimer in solution. The structure of the NAG binding site, in combination with mutagenesis studies, provide insights into the catalytic mechanism. We also show that native NAGS from human and mouse exists in tetrameric form, similar to those of bifunctional NAGS/K.
\end{abstract}

Citation: Zhao G, Jin Z, Allewell NM, Tuchman M, Shi D (2013) Crystal Structure of the N-Acetyltransferase Domain of Human N-Acetyl-L-Glutamate Synthase in Complex with N-Acetyl-L-Glutamate Provides Insights into Its Catalytic and Regulatory Mechanisms. PLoS ONE 8(7): e70369. doi:10.1371/journal.pone.0070369

Editor: Michael D.W. Griffin, University of Melbourne, Australia

Received April 12, 2013; Accepted June 17, 2013; Published July 24, 2013

This is an open-access article, free of all copyright, and may be freely reproduced, distributed, transmitted, modified, built upon, or otherwise used by anyone for any lawful purpose. The work is made available under the Creative Commons CCO public domain dedication.

Funding: This work was supported by Public Health Service grants DK-DK064913 (MT) from the National Institute of Diabetes, Digestive and Kidney Diseases. Use of the Advanced Photon Source was supported by the U. S. Department of Energy, Office of Science and Office of Basic Energy Sciences, under Contract No. W-31109-Eng-38. The funders had no role in study design, data collection and analysis, decision to publish, or preparation of the manuscript.

Competing Interests: The authors have declared that no competing interests exist.

*E-mail: dshi@cnmcresearch.org

\section{Introduction}

$\mathcal{N}$-acetylglutamate synthase (NAGS, EC 2.3.1.1) catalyzes the conversion of AcCoA and glutamate to $\mathrm{CoA}$ and $\mathcal{N}$-acetylglutamate (NAG). In microorganisms and plants, NAG is further converted to NAG phosphate by NAG kinase (NAGK, EG 2.7.2.8) to continue the L-arginine biosynthetic pathway [1,2]. However, in mammals, NAG has an entirely different role as the essential cofactor for carbamyl phosphate synthetase I (CPSI) in the urea cycle [3]. Perhaps because NAG plays different roles in lower organisms and mammals, L-arginine has opposing regulatory effects on their NAGS enzymes. In bacteria, particularly those that use the linear pathway for L-arginine biosynthesis, NAGS is feedback inhibited by the end product, L-arginine. Conversely, in mammals, L-arginine enhances the NAGS activity [3].

Phylogenetic analysis of NAGS protein sequences classifies them into two distinct types: bacteria-like, classic NAGS and vertebratelike NAGS [4]. Most bacterial and plant NAGS belong to the former, with high sequence similarity to Escherichia coli NAGS. The second type includes not only vertebrate NAGS, but also fungal NAGS and NAGK, and bacterial bifunctional NAGS/K. Nevertheless, in spite of structural similarities of the second type
NAGS of various species, it is still inhibited by L-arginine in microorganisms that utilize it.

Previously, we determined the structure of NAGS from $\mathcal{N}$. gonorrhoeae (ngNAGS) and showed that this type of NAGS has a hexameric quaternary structure and that each subunit has two distinct domains: an $\mathcal{N}$-terminal amino acid kinase (AAK) domain and a $C$-terminal $\mathcal{N}$-acetyltransferase (NAT) domain [5]. The AAK domain has a structure similar to those of various $\mathcal{N}$-acetylglutamate kinases (NAGK), but it is devoid of NAGK activity. It also has an L-arginine binding site similar to those in L-arginine sensitive NAGK structures [6]. The NAT domain has a typical GCN5-related NAT fold and a site that catalyzes NAG synthesis which is located $>25 \AA$ away from the L-arginine binding site [7]. We have also previously determined the structures of bifunctional NAGS/K from Maricaulis maris (mmNAGS/K) and Xanthomonas campestris (xcNAGS/K) [8]. Surprisingly, bifunctional NAGS/K oligomerizes to form a novel tetramer. Although the subunits of NAGS/K have similar structures to ngNAGS subunits with two distinct domains, their domain-domain linkers and relative domain orientations are different from those of ngNAGS. Inhibition by L-arginine of NAGS/K was proposed to result from 
changes in the relative orientations of AAK and NAT domains that close the AcCoA binding site.

Even though extensive efforts have been made to determine the mammalian NAGS structure, it has proven challenging because the complete protein is unstable in solution. We succeeded in obtaining stable and functional human NAGS NAT domain (hNAT) (residues 377-534) with NAG bound at 2.1 A resolution. This structure and related mutagenesis experiments allowed us to define the catalytic mechanism. We have also confirmed by crosslinking and gel-filtration experiments that both human and mouse NAGS have tetrameric oligomeric structures similar to bifunctional NAGS/K. Therefore, the mechanisms that L-arginine uses to activate mammalian NAGS and inhibit bifunctional NAGS/K may be similar despite its disparate effects on the catalytic function.

\section{Results and Discussion}

\section{Enzymatic Activity of the NAT Domain}

hNAT has detectable NAGS activity with a $V_{\max }$ value of $1.19 \pm 0.08 \mu \mathrm{mol} / \mathrm{min} / \mathrm{mg}$, but this value is approximately 6.6 fold lower than the specific activity of the full-length wild type hNAGS in the absence of L-arginine and 12.6 fold lower than the same in the presence of L-arginine $(1 \mathrm{mM})$ under similar buffer conditions [9]. AcCoA and L-glutamate titration experiments (Figure 1) indicate that the absence of the AAK domain affects AcCoA binding affinity so that hNAT has a slightly higher apparent $K_{\mathrm{m}}$ value of $1.23 \pm 0.05 \mathrm{mM}$ than the complete protein $(0.94 \pm 0.04 \mathrm{mM})$. Glutamate binding appears to be stronger, with a $K_{\mathrm{m}}$ value of $1.18 \pm 0.03 \mathrm{mM}$ lower than that of the complete protein $(2.50 \pm 0.15 \mathrm{mM})$ in the absence of L-arginine, but close to that of $1.49 \pm 0.04 \mathrm{mM}$ in the presence of L-arginine. AcCoA binding for hNAT shows significantly cooperativity with a Hill coefficient of $1.9 \pm 0.2$, in contrast to the complete hNAGS which shows no cooperativity [9].

\section{Oligomerization State in Solution}

To determine the states of oligomerization of both complete NAGS and the NAT domain in solution, cross-linking and analytic gel filtration experiments were performed. Cross-linking experiments using dimethyl suberimidate or suberic acid bis(3sulfo- $\mathcal{N}$-hydroxysuccinimide ester) sodium salt showed at least four bands on SDS-PAGE gels for both human and mouse complete NAGS, with molecular weights corresponding to oligomers of 1,2 , 3 and 4 subunits (Figure 2). Gel filtration experiments also demonstrated that complete hNAGS and mNAGS exist primarily as tetramers in solution. The molecular weights of mNAGS and hNAGS calculated from the standard curve are 199.2 and 220.1 $\mathrm{KDa}$, respectively, consistent with tetramer molecular weights of 195.8 and 202.4 KDa for mNAGS and hNAGS, respectively. Molecular weights of mNAT and hNAT calculated from the standard curve are 36.2 and $36.1 \mathrm{kDa}$, respectively, implying they exist as dimers in solution since molecular weights of mNAT and hNAT dimers calculated based on the expected amino acid sequenced are $36.1 \mathrm{kDa}$ matching the observed weight. The results are consistent with those for bifunctional mmNAGS/K and xcNAGS/K and imply that the hNAGS and mNAGS have similar tetrameric architectures to mmNAGS/K and $\mathrm{xcNAGS} / \mathrm{K}$ in solution.

\section{Structure of hNAT with NAG Bound}

The structure of hNAT (residue 377 to 534) was determined at $2.1 \AA$ resolution and refined to $R_{\text {work }}$ and $R_{\text {free }}$ values of $18.4 \%$ and $24.4 \%$, respectively (Table 1 ). The model has good geometry with $92.5 \%$ of the residues located inside the most favored area of a Ramachantran plot. Four copies of each subunit were identified in the asymmetric unit. The structures of the four subunits were not defined equally well with subunit A best defined, followed by subunit $\mathrm{X}$, subunit $\mathrm{B}$ and subunit $\mathrm{Y}$, with average temperature $B$ factors of $35.0 \AA^{2}, 44.9 \AA^{2}, 54.2 \AA^{2}$ and $78.1 \AA^{2}$, respectively. Superimpositions of the four subunits result in RMS deviations of 0.4-0.8 $\AA$ (Table 2) with subunits $\mathrm{A}$ and $\mathrm{B}$ most similar, and subunit $\mathrm{A}$ and $\mathrm{X}$ most different. As shown in Figure 3B, the core secondary structures are very similar for all subunits, with the major differences in loop regions and terminal residues, which are usually highly flexible and easily affected by the different packing environments in the crystal. Since the structure of subunit A has the best quality, the structure description and discussion will be mainly based on this subunit.
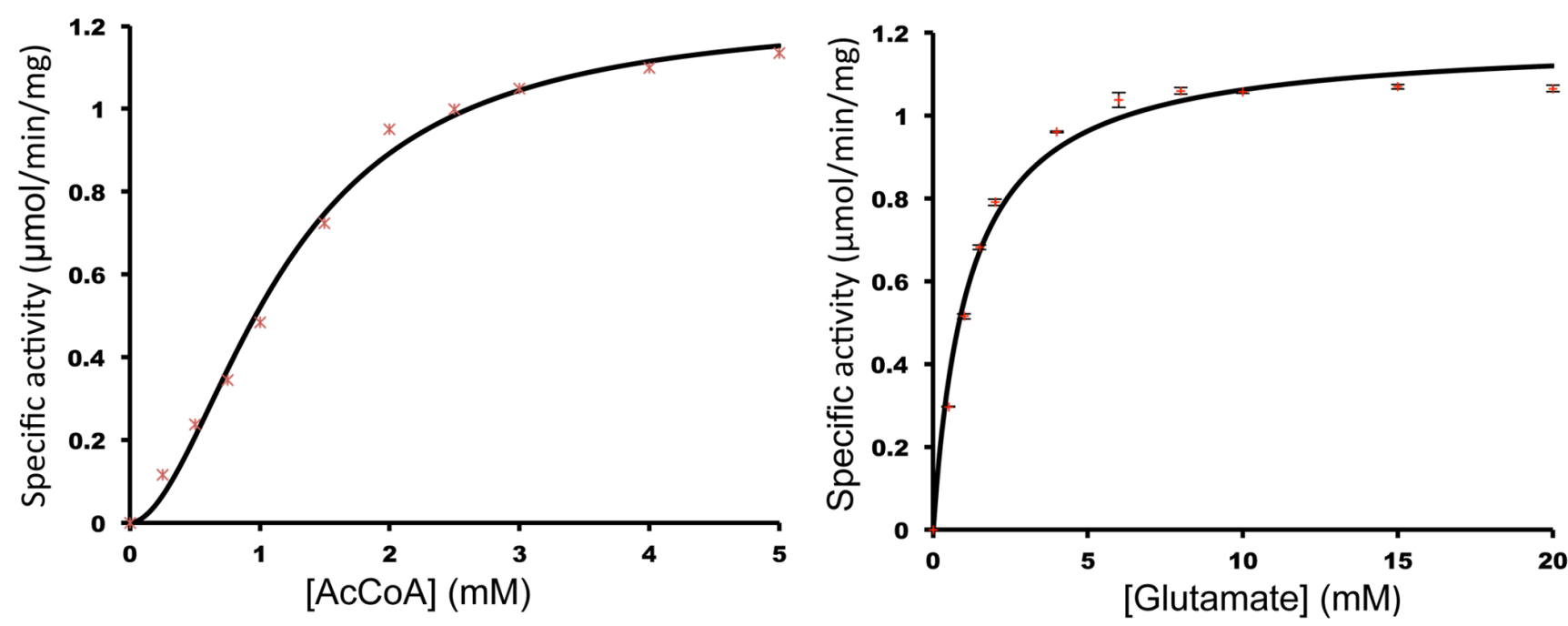

Figure 1. Biochemical properties of hNAT. A and B, dependence of enzyme activity on the concentration of AcCoA or L-glutamate. AcCoA or Lglutamate was varied in the range of $0.25-5.0$ and $0.50-20.0$, respectively, with L-glutamate and AcCoA fixed at 10 or $2.5 \mathrm{mM}$, respectively. doi:10.1371/journal.pone.0070369.g001 
A

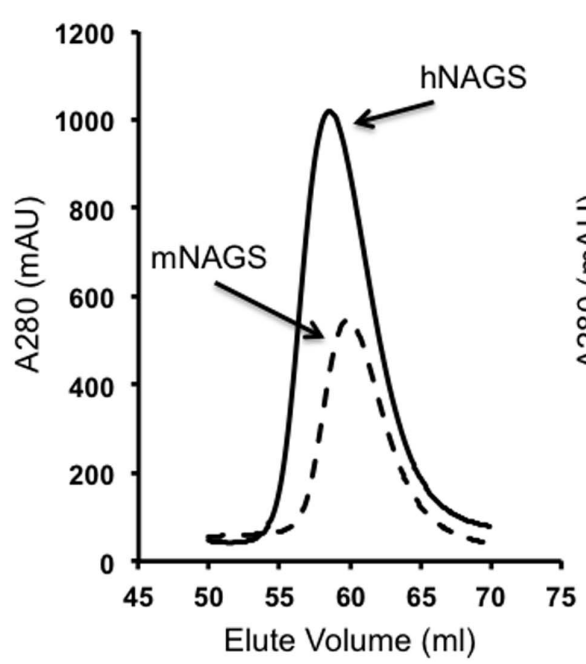

B
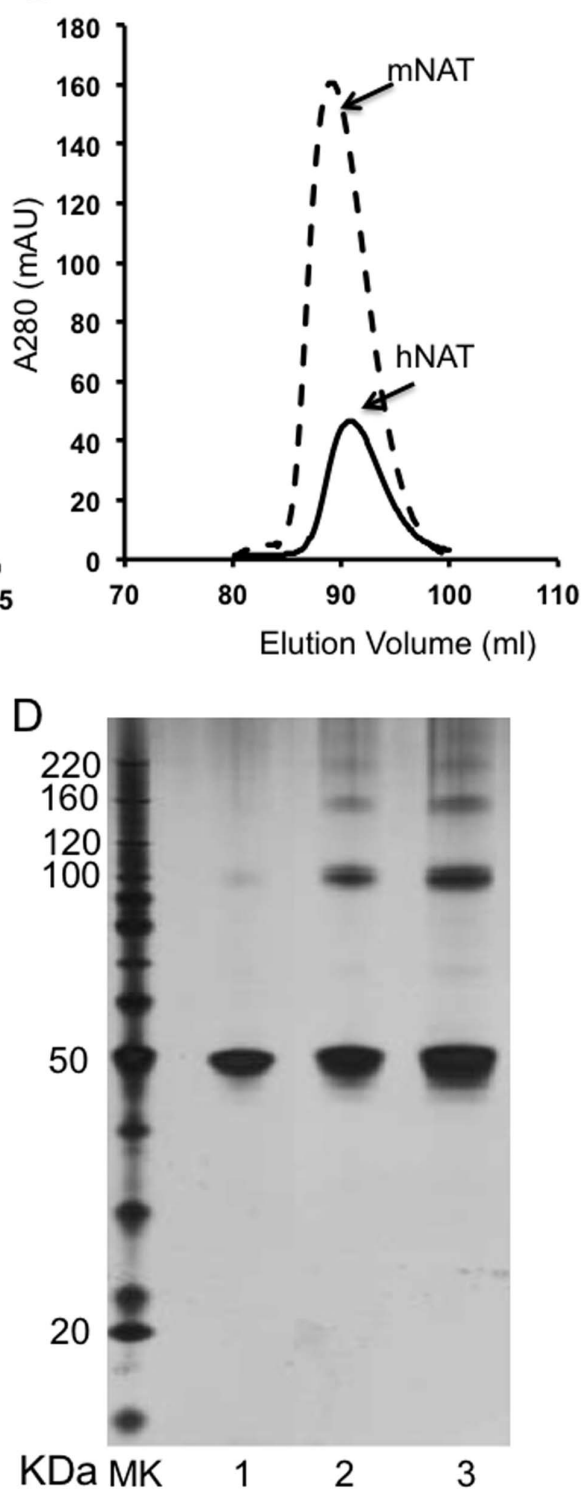

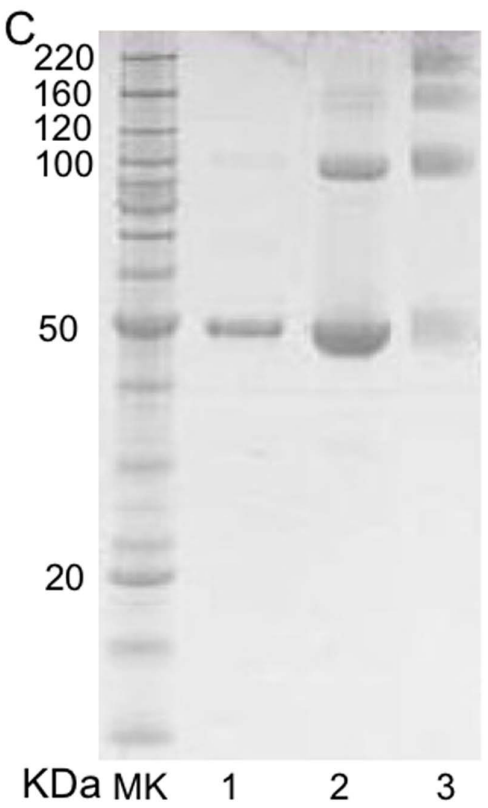

Figure 2. Oligomeric structure of mNAGS and hNAGS in solution. A: Analytic gel chromatography of mNAGS and hNAGS. Elution profiles of mNAGS and hNAGS are shown in dashed and solid lines, respectively. B: Analytic gel chromatography of mNAT and hNAT. Elution profiles of mNAT and hNAT are shown in dashed and solid lines, respectively. C: Cross-linking of mNAGS. Lanes 1; protein size markers; 2, mNAGS (2.5 $\mu \mathrm{g})$ without cross-linking reagent; 3 , mNAGS $(2.5 \mu \mathrm{g})$ with cross-linking reagent, suberic acid bis(3-sulfo-N-hydroxysuccinimide ester) sodium salt; 4 , mNAGS with cross-linking reagent, dimethyl suberimidate dihydrochloride. D: Cross-linking of mNAGS. Lanes 1; protein size markers; 2 , mNAGS without crosslinking reagent; 3 , mNAGS $(1.5 \mu \mathrm{g})$ with cross-linking reagent, dimethyl suberimidate dihydrochloride; 4 , mNAGS $(4.5 \mu \mathrm{g})$ with cross-linking reagent, dimethyl suberimidate dihydrochloride. doi:10.1371/journal.pone.0070369.g002

Each subunit has a central seven-strand $\beta$-sheet arranged as a $V$ shaped structure with three anti-parallel $\beta$-strands in the $C$ terminal arm and four anti-parallel $\beta$-strands in the $\mathcal{N}$-terminal arm (Figure 3A). The central $\beta$-sheet is flanked by five helices with three helices on one side and four helices on the other. The structure has a typical fold of GCN5-related $\mathcal{N}$-acetyltransferase and is similar to the NAT domain structure of the bifunctional NAGS/K from $M$. maris (Table 2).

\section{Dimerization}

Even though four subunits were identified in an asymmetric unit, the PISA server [10] indicated that the stable molecule is dimer. Subunit A and subunit B form a molecular dimer. The molecular dimers for subunit $\mathrm{X}$ and subunit $\mathrm{Y}$ were generated via crystallographic two-fold symmetries, respectively. At each dimer interface (A-B, X-X or Y-Y), the $C$-terminal arm from one subunit interacts with the $\mathrm{C}$-terminal arm from the other subunit to form a continuous 6 -strand antiparallel $\beta$-sheet, similar to the NAT-NAT domain interaction observed in the mmNAGS/K structure (Figure 3G). This extensive interface has a buried interface of $1477 \AA^{2}$. The interactions in this interface involve extensive mainchain (Asp490, Ser492 and Ser494) and side-chain (Ser494) hydrogen bonding interactions, $\pi-\pi$ interactions (Phe496- 
Table 1. Data collection and refinement statistics.

\begin{tabular}{|c|c|}
\hline \multicolumn{2}{|l|}{ Data collection } \\
\hline Bound ligands & NAG \\
\hline Space group & $P 4_{3} 2_{1} 2$ \\
\hline Wavelength ( $(\AA)$ & 1.0 \\
\hline Resolution $(\AA ̊)$ & $50-2.10(2.14-2.10)^{\mathrm{a}}$ \\
\hline \multirow[t]{2}{*}{ Unit-cell parameters $(\AA)$} & $a=b=116.1$ \\
\hline & $c=109.7$ \\
\hline Measurements & 600,087 \\
\hline Unique reflections & $83,202(4,107)$ \\
\hline Redundancy & $7.2(5.1)$ \\
\hline Completeness (\%) & $99.9(99.1)$ \\
\hline$<l / \sigma(l)>$ & $21.7(1.7)$ \\
\hline$R_{\text {merg }}(\%)^{\mathrm{b}}$ & $8.1(65.6)$ \\
\hline \multicolumn{2}{|l|}{ Refinement } \\
\hline Resolution range $(\AA)$ & $40-2.10(2.15-2.10)$ \\
\hline No. of protein atoms & 4,963 \\
\hline No. of water atoms & 266 \\
\hline No. of hetero atoms & 52 \\
\hline Rmsd of bond lengths $(\AA)$ & 0.008 \\
\hline Rmsd of bond angle $\left({ }^{\circ}\right)$ & 1.1 \\
\hline$R_{\text {work }}(\%)^{c}$ & $18.5(25.2)$ \\
\hline$R_{\text {free }}(\%)^{d}$ & $24.4(31.5)$ \\
\hline \multicolumn{2}{|l|}{ Ramachandran plot (\%) } \\
\hline Favored & 92.5 \\
\hline Allowed & 7.3 \\
\hline Generous & 0.2 \\
\hline Disallowed & 0.0 \\
\hline
\end{tabular}

${ }^{\mathrm{a}}$ Figures in brackets apply to the highest-resolution shell.

${ }^{\mathrm{b}} R_{\text {merg }}=\Sigma_{h} \Sigma_{i}|/(h, i)-<I(h)>| / \Sigma_{h} \Sigma_{j} l(h, i)$, where $l(h, i)$ is the intensity of the $i$ th observation of reflection $h$, and $\langle I(h)\rangle$ is the average intensity of redundant measurements of reflection $h$.

${ }^{c} R_{\text {work }}=\Sigma_{h}|| F_{\text {obs }}|-| F_{\text {calc }}|| / \Sigma_{h}\left|F_{\text {obs }}\right|$.

${ }^{\mathrm{d}} R_{\text {free }}=\Sigma_{h}|| F_{\text {obs }}|-| F_{\text {calc }}|| \Sigma_{h}\left|F_{\text {obs }}\right|$ for $5 \%$ of the reserved reflections. doi:10.1371/journal.pone.0070369.t001

Phe496'), $\pi$-cation interactions (Tyr512-Arg509') and other hydrophobic interactions (Figure 3D).

\section{NAG Binding Site}

The electron density map was readily interpretable with NAG visible at the enzyme active site. NAG binds in a cavity surrounded by the central $\beta$-sheet (strands $\beta 16$ and $\beta 17$ ), the loop connecting helices $\alpha 11$ and $\alpha 12$, and the $C$-terminal segment (Figure 3A). The side-chains of five residues, Lys444 from the strand $\beta 16, \operatorname{Arg} 474$ and Arg476 from strand $\beta 17$, Asn 479 from the loop connecting $\beta 17$ and $\alpha 14$ and Lys401 from the loop connecting helices $\alpha 11$ and $\alpha 12$ are involved in hydrogen bonding to NAG (Figure 4A, Table 3). The main-chain O of Asp443 and Arg473 and the mainchain N of Phe445 and Arg476 are also involved in positioning NAG by anchoring different functional groups of NAG. The sidechains of Phe399, Leu442, Trp498 and Phe525 form hydrophobic interactions with the side-chain of NAG holding the side-chain in place. These extensive hydrogen bonding and hydrophobic interactions place NAG or L-glutamate in the right position and orientation to facilitate the catalytic reaction and define the specificity of hNAGS. All these residues are either invariant
Table 2. RMSD values $(\AA)$ among different subunit within NAG bound structure and with the bifunctional mmNAGS/K native structure.

\begin{tabular}{lllll}
\hline & & & & \\
\hline Subunit & $\mathbf{A}$ & $\mathbf{B}$ & $\mathbf{X}$ & $\mathbf{Y}$ \\
\hline A & $\mathbf{1 . 0 8 ^ { \mathbf { a } }}$ & 0.42 & 0.76 & 0.60 \\
$\mathrm{~B}$ & $\mathbf{1 . 1 9}$ & 0.69 & 0.61 \\
$\mathbf{X}$ & $\mathbf{1 . 0 5}$ & & 0.48 \\
Y & $\mathbf{1 . 0 4}$ & & \\
\hline $\begin{array}{l}\text { aThe values in bold are RMSD of the subunit A in hNAT structure with subunits } \\
\text { in mmNAGS/K NAT domain structure (PDB 3S6H). } \\
\text { doi:10.1371/journal.pone.0070369.t002 }\end{array}$ &
\end{tabular}

(Phe399, Leu442, Asp443, Lys444, Phe445, Arg474, Arg476, Asn479, Trp498 and) or conservatively substituted (Lys401 and Phe525) in vertebrate-like NAGS. However, in contrast to bacterial-like NAGS such as Neisseria gonorrhoeae NAGS [5], hNAGS uses different residues to bind NAG, supporting the hypothesis that the NAT domains of vertebrate-like and bacteriallike NAGS evolved from different ancestors.

\section{CoA Binding Site}

Even though $10 \mathrm{mM} \mathrm{CoA}$ was present in the crystallization solution, no continuous electron density corresponding to CoA was observed in the " $V$-shaped" groove where the pantetheine moiety of CoA usually binds, probably due to suboptimal conditions for CoA binding. However, the unambiguous identification of NAG in the expected site suggests that CoA is likely to bind in a site similar to those found in other GCN5-related acetyltransferases. Structural comparison of hNAT with the NAT domain of ngNAGS, which has both substrates bound, allows identification of the CoA binding site and development of a model of the catalytic mechanism. Superimposition of the hNAT with the NAT domain of ngNAGS clearly indicates that the pantetheine moiety of CoA interacts with the protein through hydrogen bonds with the main-chain nitrogen of Phe 445 and the main-chain oxygen of Val447 from $\beta 16$, in a way similar to an anti-parallel $\beta$ sheet (Figure 5). The thiol sulfur is oriented in a position within hydrogen bonding range of the side-chains of Tyr495 and Ser475. One water molecule (w37) was identified occupying the thiol sulfur position in the present structure, $3.4 \AA$ away from the acetyl carbon and perpendicular to the acetyl group plane of NAG. The structure is consistent with hNAGS using a one-step direct attack catalytic mechanism to transfer the acetyl group from AcCoA to the amino group of L-glutamate, as is the case for most members of the GCN5-related NAT family. The pyrophosphate moiety appears to be in proximity to the sequence Gln452-Gly453Gln454-Gly455-Ser456-Gly457-Gln458, which conforms to (Arg/Gln)-Xaa-Xaa-Gly-Xaa-(Gly/Ala) motif for AcCoA recognition and binding in known GCN5-related $\mathcal{N}$-acetyltransferases [11]. Because of the absence of AcCoA or CoA binding, this part of structure varied significantly among different subunits (Figure 3B). Upon AcCoA or CoA binding, the structure needs to adjust in order to bind the pyrophosphate moiety. The sidechains of Gln454 and Gln458 of this motif, as well as Trp484, seem to be involved in positioning CoA. The adenosine moiety of CoA is located on the surface of the protein, as seen in other GCN5-related NAT structures [12]. 

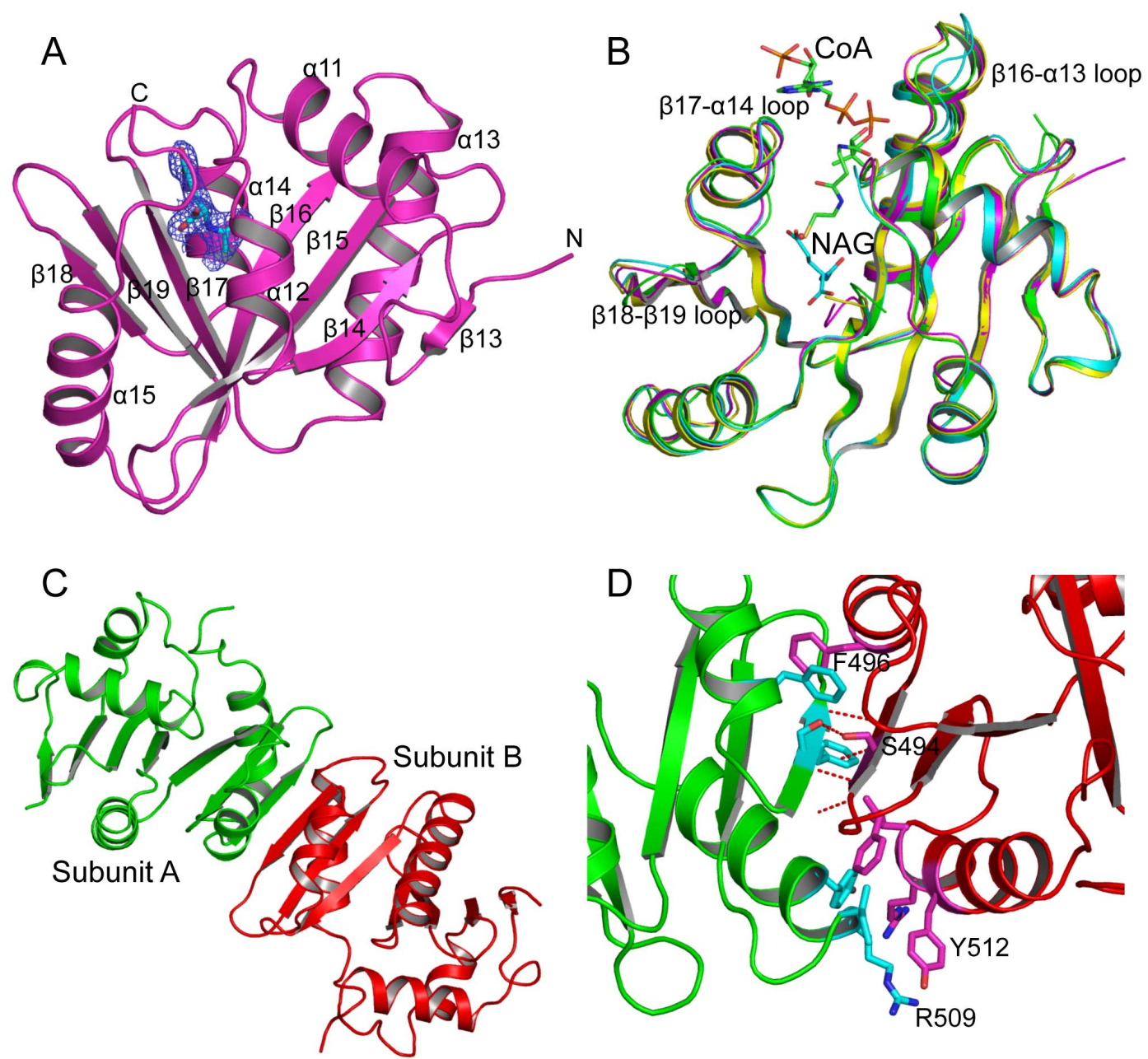

Figure 3. Structure of hNAT. A: Ribbon diagram of hNAT subunit structure. Bound NAG is shown as sky-blue sticks. The electron density map $\left(2 \mathrm{~F}_{\mathrm{o}}-\mathrm{F}_{\mathrm{c}}\right.$ ) around bound NAG (contoured at $\left.1.0 \sigma\right)$ is shown as blue cage. B: Superimposition of four hNAT subunits in asymmetric unit. The bound NAG is shown as sky-blue sticks. The proposed bound CoA is shown as green sticks. Subunits $A, B, X$ and $Y$ are shown in pink, yellow, green and blue ribbons, respectively. C: The hNAT molecular dimer. Subunits A and B are shown in green and red ribbons, respectively. D: Details of the interactions between subunits $A$ and B. Side-chains of the residues in the interface are shown in sticks. Potential hydrogen bonding interactions are shown in red dashed lines.

doi:10.1371/journal.pone.0070369.g003

\section{Comparison of the NAT Domain Structures of Human NAGS and mmNAGS/K}

The overall hNAT structure is similar to that of mmNAGS/K (Figure 6, Table 2) and can be aligned with an RMS deviation of $\sim 1.0 \AA$, even though different subunits in mmNAGS/K have different relative orientations of the AAK and NAT domains [8]. The major structural differences occur in the loop regions $(\alpha 12-$ $\beta 14, \beta 16-\alpha 13, \beta 15-\beta 16$ and $\beta 19-\alpha 15$ loops). The significant conformational changes in the pyrophosphate moiety binding motif in the loop connecting $\beta 16$ and $\alpha 13$ demonstrate the high flexibility in this region in the absence of AcCoA binding, as shown in the variation among different subunits. The conformational changes of the side-chain position of Arg476 may be functionally significant. In all mmNAGS/K subunits, the side-chain of Arg388 (the equivalent residue of Arg476) points outwards (Figure 6) whereas in the NAG bound hNAT structure, this side-chain moves towards the substrate binding site to anchor the $\gamma$-carboxyl group of NAG. Another interesting difference is in the $\alpha 12-\beta 14$ loop in which two more residues are present in hNAT compared to mmNAGS/K. The side-chain of Arg414 of this loop swings towards the NAG binding site to form a hydrogen bond with the side-chains of Asp433 and Asp443. At least 8 nearby water molecules link the amino nitrogen of NAG to the side-chains of Tyr441, Asp443, Lys444, Ser524, Arg414 and Ser410 in a string that extends to the protein surface (Figure 4B). As proposed for serotonin $\mathcal{N}$-acetyltransferase [13], this chain of water molecules may be a "proton wire" to ferry away a proton from the substrate and facilitate a nucleophilic attack on AcCoA.

\section{Implications for Catalysis}

To confirm the catalytic mechanism, several residues in this site were selected for biochemical studies. Tyr485, the equivalent residue of Tyr397 in mmNAGS/K and Tyr405 in xcNAGS/K, appears to act as a catalytic acid that donates a proton to the thiol group of CoA, playing an important role in the catalytic reaction (Figure 4A). This equivalent tyrosine could be identified in most GCN5-related acetyltransferases [14]. Indeed, the Y485F mutant showed 10 fold lower catalytic activity than wild-type protein (Table 4). 
Table 3. Interactions between $N$-acetyl-L-glutamate and protein atoms.

\begin{tabular}{|c|c|c|c|c|c|}
\hline \multirow{2}{*}{ Arginine } & \multirow{2}{*}{ Protein } & \multicolumn{4}{|c|}{ Distance (Å) } \\
\hline & & Subunit $A$ & Subunit B & Subunit $X$ & $X$ Subunit $Y$ \\
\hline \multirow[t]{2}{*}{ N2 } & Asp443 O & 3.37 & 3.41 & 3.29 & 3.29 \\
\hline & Arg474 O & 3.23 & 3.19 & 3.23 & 3.33 \\
\hline 07 & Phe445 N & 2.96 & 3.00 & 3.04 & 3.24 \\
\hline \multirow[t]{2}{*}{ OXT } & Lys444 NZ & 3.08 & 2.61 & 2.97 & 3.46 \\
\hline & Wat $258^{\mathrm{a}} O$ & 2.47 & 3.37 & & \\
\hline \multirow[t]{3}{*}{ O } & Arg474 NE & 2.94 & 3.16 & 2.96 & 2.87 \\
\hline & Wat258 O & 3.22 & 2.47 & 2.47 & \\
\hline & Wat9 O & 2.64 & & & \\
\hline \multirow[t]{2}{*}{ OE1 } & Asn479 ND & 2.96 & 3.48 & $4.95^{\mathrm{b}}$ & 3.43 \\
\hline & Arg476 N & 2.98 & 3.10 & $4.22^{\mathrm{b}}$ & 3.19 \\
\hline \multirow[t]{2}{*}{ OE2 } & Lys401 NZ & 2.64 & 3.31 & 2.28 & $4.01^{b}$ \\
\hline & Arg476 NE & 2.61 & 3.69 & 2.77 & $3.53^{b}$ \\
\hline
\end{tabular}

${ }^{a}$ Water numbering for subunit $A$ only.

${ }^{b}$ The distances are too far away for hydrogen bonding interactions. doi:10.1371/journal.pone.0070369.t003

Since the $\alpha$-amino group of $\mathrm{L}$-glutamate has a $\mathrm{pKa}$ value that is close to 10 , it seems clear that amine deprotonation must precede the acetyl group transfer. The highly conserved Tyr441 located in the water channel that connects to the $\alpha$-amino group (see previous section), is positioned to play a role as the catalytic base in proton removal. The lower activity of Y441F mutant is consistent with this catalytic role of this tyrosine. The 7 fold lower activity for N479A mutant confirmed that it is a key residue to bind Lglutamate as found in the present structure (Figure 4A).

\section{Mechanism of L-arginine Regulation}

Since hNAGS and mNAGS have similar oligomeric structures (tetramers), as demonstrated in our cross-linking and gel-filtration experiments (Figure 2), and the dimer architecture of hNAT is similar to the NAT-NAT domain interface in mmNAGS/K (Figure $3 \mathrm{C}$ ), the quaternary structure of hNAGS and mNAGS is likely to be similar to that of bifunctional mmNAGS/K. Larginine binding may also cause rotation of the NAT domain towards to the AAK domain in mammalian NAGS, but to a lesser degree than in mmNAGS/K to allow AcCoA to bind to the active site, because the domain linkers of mammalian NAGS and bacterial bifunctional NAGS/K consist of different amino acids. The enhancement of NAGS activity by arginine in mammalian NAGS may be caused by increasing the AcCoA binding affinity via favorable hydrogen bonding interactions of residues in the AAK domain, facilitated by the conformational changes induced upon arginine binding.

\section{Roles for the AAK Domain}

The major role of NAGS in the urea cycle is to produce the essential cofactor, NAG, to activate CPSI. Among the three mitochondrial enzymes of the urea cycle, NAGS is the least abundant by far, thousands fold lower than CPSI and OTCase. Since the NAT domain alone has catalytic activity and is stable, an interesting question arises: why has the AAK domain remained intact through evolution? Even though activity assays demonstrate that the AAK domain enhances NAGS activity 6 to 12 fold, this may not be the major reason since an increase in enzyme abundance could compensate for lower activity. A more probable explanation is a regulatory role of the AAK domain in urea cycle flux. Complete hNAGS has two extra features relative to hNAT that may play a role in regulating urea cycle flux. First, the binding of L-arginine enhances NAGS activity and the arginine-binding site that is located in the AAK domain is conserved in NAGS across phyla [4]. In microorganisms, arginine biosynthesis is regulated via this arginine binding site because bound L-arginine is an allosteric inhibitor of NAGS activity [7]. It is therefore reasonable to assume that in mammals, urea cycle flux can be rapidly enhanced via increased NAGS activity by L-arginine binding at this site. Our $\mathcal{N}$-carbamylglutamate (NCG) clinical trial experiments demonstrated that NCG could enhance urea cycle flux even in healthy individuals [15], implying that under normal conditions, CPSI is not fully saturated with NAG. Increasing NAG production will therefore increase urea production by activating additional CPSI molecules. Second, the presence of a proline-rich region in the $\mathcal{N}$-terminal sequence of mammalian NAGS (AAK domain) may be important in interacting with CPSI to facilitate NAG translocation from NAGS to CPSI. Proline-rich motifs often serve as targets for protein recognition and interaction since they are recognized by many proteins, including important signaling proteins such as Src homology 3 [16], the WW domain of a kinase-associated protein [17], Enabled/VASP (EVH1) [18] and ubiquitin-E2-like variant (UEV) domain of the tumor maintenance protein Tsg101 [19]. Crystal structures of these motifs demonstrate that they are usually exposed to solvent and have a collagenlike polyproline type II (PPII) extended conformations. Most of these PPII motifs are involved in protein-protein interactions that seem important for signal transduction and metabolic regulation [20].

\section{Clinical Implications}

This study demonstrates that hNAT is stable and has catalytic activity. The results are consistent with previous observations about potential effects of hNAGS missense mutations in patients. Missense mutations in the AAK domain are usually "milder" than mutations in the NAT domain and are usually associated with "late-onset" clinical presentation [21]. All missense mutations associated with neonatal-onset, severe manifestations identified so far are located in the NAT domain. While the NAT domain plays a key role in NAGS activity and is mainly encoded by the last three exons of the human gene, the mitochondrial peptide signal and the proline-rich variable segment are encoded by the first exon of the gene [22]. Thus, a putative nonsense and out of frame mutations in the AAK domain (exons 2-4) might be rescued by exon skipping therapy that could restore the correct reading frame for encoding the NAT domain.

\section{Materials and Methods}

\section{Cloning and Protein Expression and Purification}

Human NAGS (hNAGS), mouse NAGS (mNAGS), hNAT and all mutants were expressed and purified as described previously [5]. Briefly, the proteins were expressed in E. coli BL21(DE3) cells (Invitrogen) and purified with nickel affinity and Histrap SP columns (GE Healthcare). Protein purity was verified by SDS/ PAGE gel and protein concentration was measured with a Nanodrop 1000 spectrophotometer (Thermo Scientific). The extinction coefficient obtained from the ExPASy web server (http://web. expasy.org/protparam/) was used to calculate protein concentrations. The protein was stored at $253 \mathrm{~K}$ in a buffer containing $50 \mathrm{mM}$ Tris-HCl, pH 7.4, $50 \mathrm{mM} \mathrm{NaCl}, 10 \%$ glycerol, $5 \mathrm{mM} \beta$ mercaptoethanol, and $1 \mathrm{mM}$ EDTA. 
A

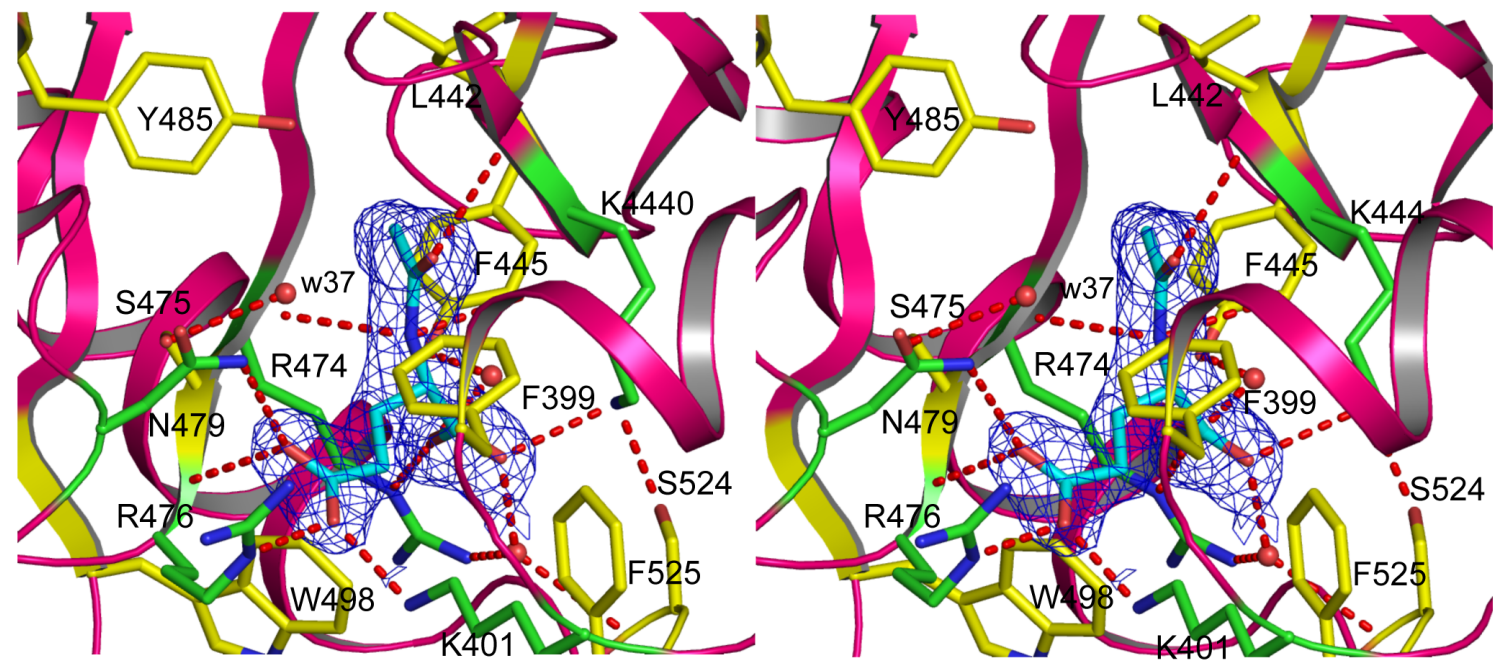

$\mathrm{B}$

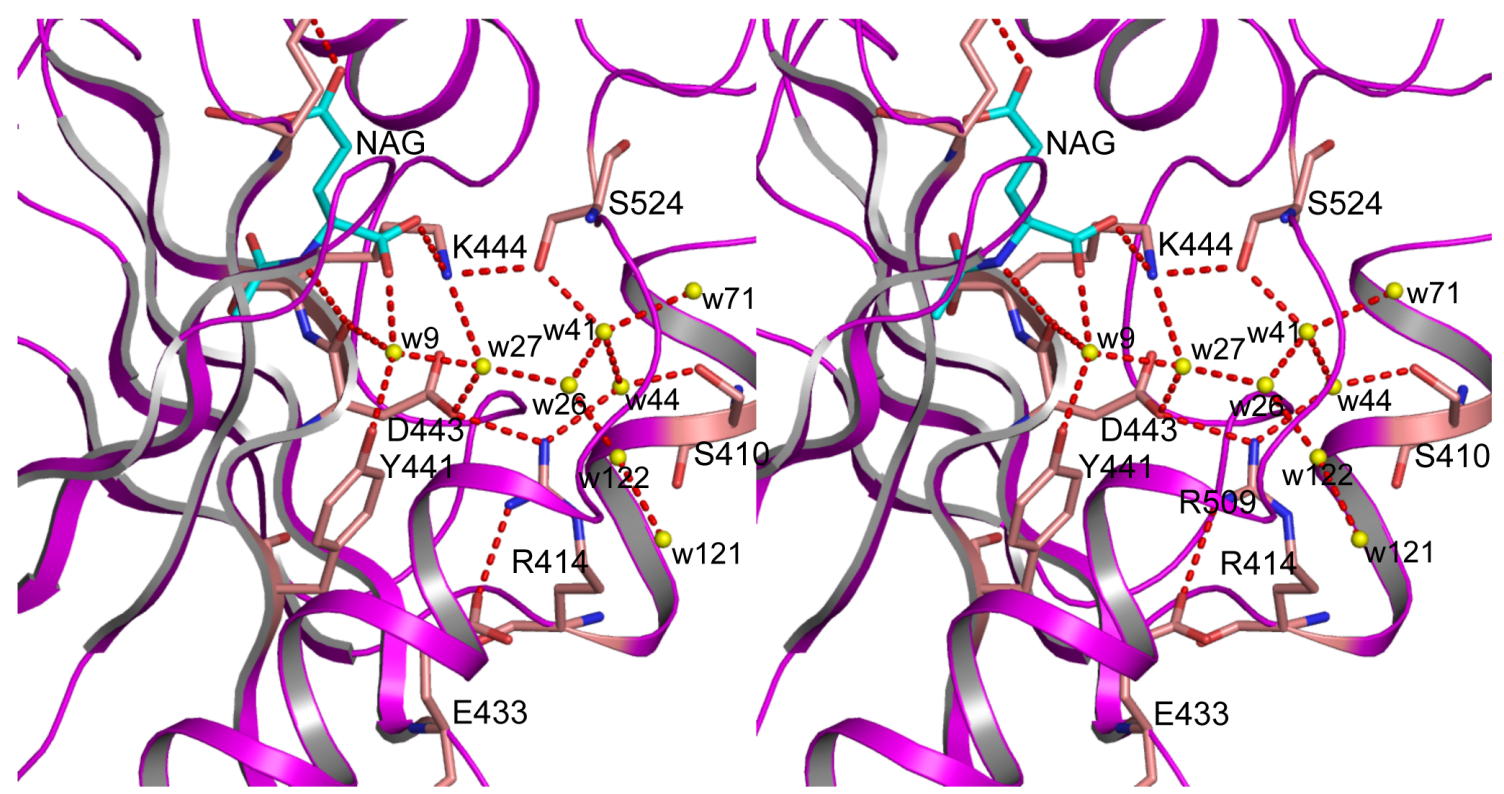

Figure 4. NAG binding site. A: Stereo diagram of NAG binding site. The bound NAG is shown in sky-blue sticks. The side-chains involved in hydrogen bonding interactions with NAG are shown in green sticks. The side-chains of other surrounding residues are shown in yellow sticks. The water molecule (w37) is shown in red ball. The electron density map $\left(2 \mathrm{~F}_{\mathrm{o}}-\mathrm{F}_{\mathrm{c}}\right)$ around bound NAG (contoured at $\left.1.0 \sigma\right)$ is shown as blue cage. Potential hydrogen bonding interactions are shown in red dashed lines. B: Stereo diagram of "water wire" channel. The bound NAG is shown in sky-blue sticks. Water molecules are shown in yellow balls. Residues involved in hydrogen bonding interactions are shown in brown sticks. Potential hydrogen bonding interactions are shown in red dashed lines.

doi:10.1371/journal.pone.0070369.g004

\section{Site-directed Mutagenesis}

Site-directed mutant DNA sequences encoding hNAT were created using primers containing the desired mutations and the QuikChange Mutagenesis Kit according to the manufacturer's protocol (Strategene). The sequences of mutant DNA sequences were verified by DNA sequencing.

\section{Activity Assay}

Enzymatic activity was assayed using the method described previously [23]. A stable isotope dilution method using liquid chromatography mass spectrometry (LC-MS) to measure NAG production was adapted. Each assay was performed in a $100 \mu \mathrm{l}$ solution containing $50 \mathrm{mM}$ Tris, $\mathrm{pH} 8.5,10 \mathrm{mM}$ glutamate and $2.5 \mathrm{mM}$ AcCoA. The reaction was initiated by the addition of purified recombinant enzyme $(20 \mu \mathrm{g})$, and the mixture was incubated at $303 \mathrm{~K}$ for $5 \mathrm{~min}$ and quenched with $100 \mu \mathrm{l}$ of $30 \%$ trichloroacetic acid containing $50 \mu \mathrm{g}$ of $\mathcal{N}$-acetyl- $\left[{ }^{13} \mathrm{C}_{5}\right]$ glutamate $\left({ }^{13} \mathrm{C}-\mathrm{NAG}\right)$ as an internal standard. Precipitated protein was removed by micro-centrifugation. The supernatant $(10 \mu \mathrm{l})$ was submitted to LC-MS (Agilent) analysis. The mobile phase consisted of $92 \%$ solvent A (1 ml trifluoroacetic acid in $1 \mathrm{~L}$ water) and $8 \%$ solvent $\mathrm{B}(1 \mathrm{ml}$ trifluoroacetic acid in $1 \mathrm{~L}$ of $1: 9$ water/ acetonitrile) and the flow rate was $0.6 \mathrm{ml} / \mathrm{min}$. Glutamate, NAG, 

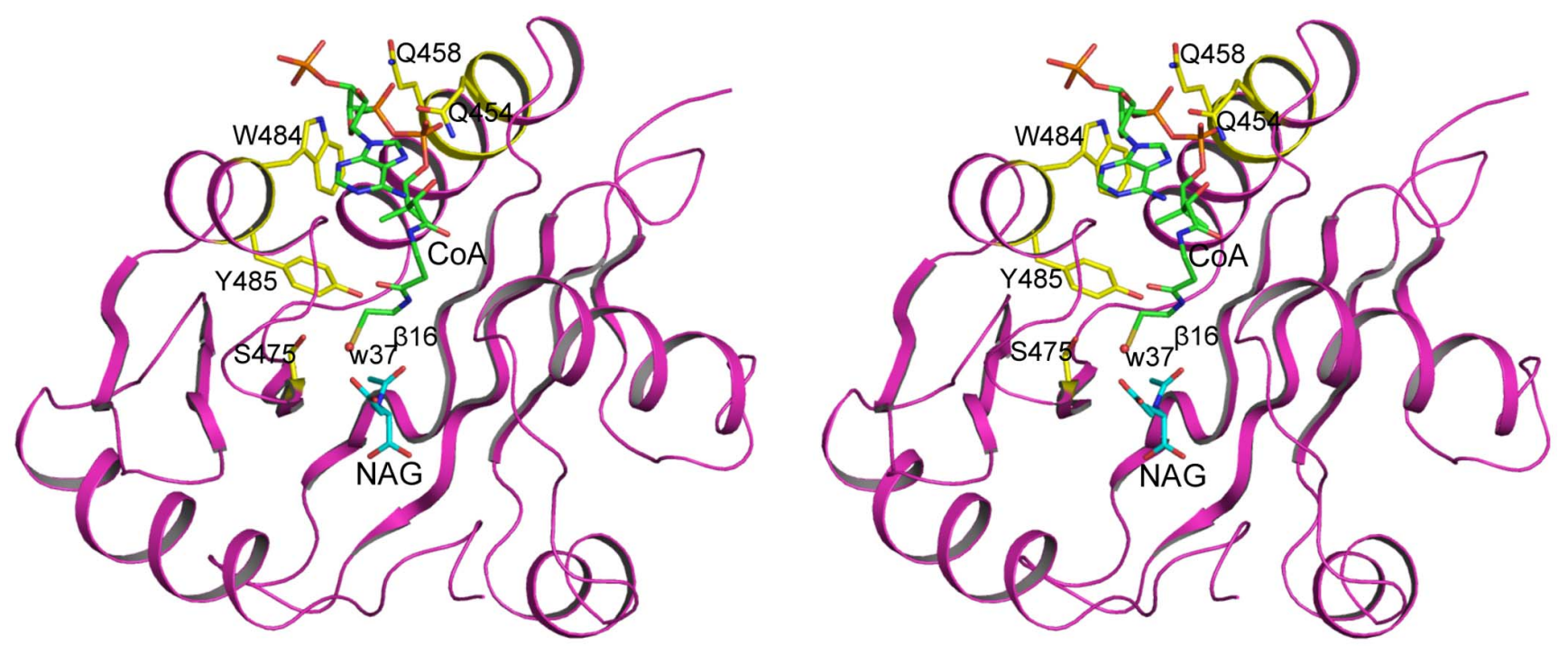

Figure 5. Stereo diagram of the proposed CoA binding site. The proposed bound CoA is shown in green sticks. The bound NAG is shown in sky-blue sticks. Side-chains of residues that potentially hydrogen bond to CoA are shown in yellow sticks. The water molecule (w37) that occupies the similar position of thiol $\mathrm{S}$ of $\mathrm{COA}$ is shown in a red ball. doi:10.1371/journal.pone.0070369.g005

and ${ }^{13} \mathrm{C}-\mathrm{NAG}$ were detected and quantified by selected ion monitoring mass spectrometry.

AcCoA and glutamate titration experiments were carried out with AcCoA or L-glutamate concentration varied in the range of $0.25-5.0$ and $0.5-20 \mathrm{mM}$, respectively, and L-glutamate or AcCoA concentration fixed at 10 and $2.5 \mathrm{mM}$, respectively. The L-glutamate titration data were fit to Michaelis-Menten kinetics, while AcCoA titration data were fit to sigmoidal kinetics $\left(V=V_{\max }\right.$ $[\mathrm{AcCoA}]^{n} /\left([\mathrm{AcCoA}]^{n}+K_{\mathrm{m}}{ }^{n}\right)$, where $V_{\max }$ is maximum activity, $K_{\mathrm{m}}$ is half-maximum activity and $n$ is the Hill coefficient, using the program GNUPLOT.

\section{Cross-linking Experiment}

Cross-linking experiments were performed using the protocol described by Davies and Stark [24]. mNAGS $(2.5 \mu \mathrm{g})$ and hNAGS $(1.5$ and $4.5 \mu \mathrm{g})$ were incubated with the cross-linking reagent dimethyl suberimidate $(4.5 \mu \mathrm{g})$ or suberic acid bis(3-sulfo$\mathcal{N}$-hydroxysuccinimide ester) sodium salt $(9.0 \mu \mathrm{g})$ in $10 \mu \mathrm{l}$ solution
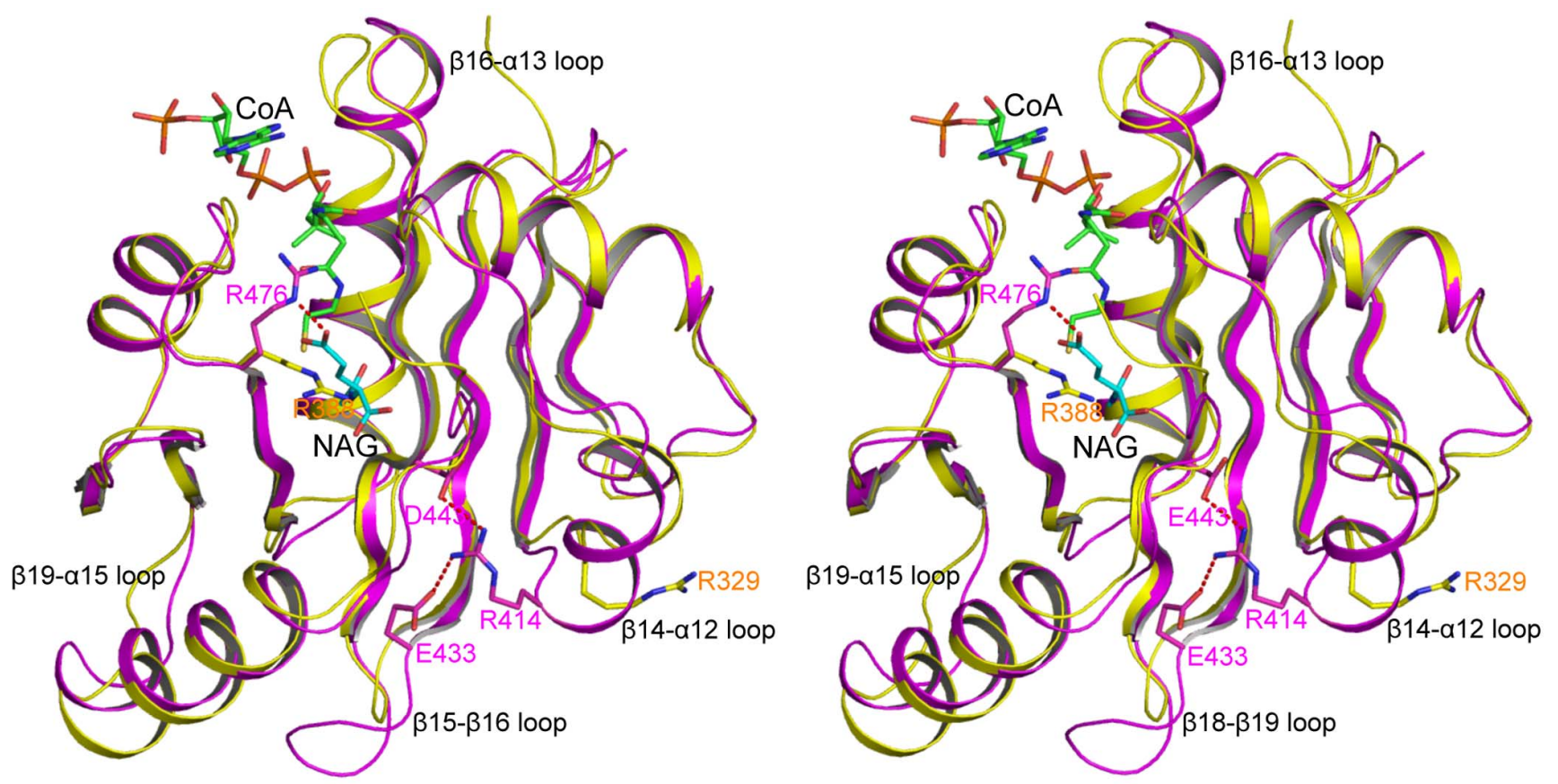

Figure 6. Superimposition of hNAT with the NAT domain of subunit $X$ of mmNAGS/K. The structure of hNAT is shown in pink ribbons. The structure of the NAT domain of subunit $\mathrm{X}$ of $\mathrm{mmNAGS/K}$ is shown in yellow ribbons. The bound NAG is shown in sky-blue sticks. The proposed bound COA is shown in green sticks. Residues that are mentioned in text are shown in sticks. doi:10.1371/journal.pone.0070369.g006 
Table 4. Enzyme activities of hNAT and active site mutants.

\begin{tabular}{ll}
\hline Sample & Activity $(\mu \text { moles } / \mathbf{m i n} / \mathbf{m g})^{\mathbf{a}}$ \\
\hline WT & $1.05 \pm 0.01$ \\
WT+L-arginine $(1 \mathrm{mM})$ & $1.04 \pm 0.01$ \\
Y485F & $0.078 \pm 0.003$ \\
Y441F & $0.857 \pm 0.004$ \\
N479A & $0.154 \pm 0.002$ \\
\hline
\end{tabular}

${ }^{\mathrm{a}}$ Means \pm standard errors of means $(n=3)$ are shown

doi:10.1371/journal.pone.0070369.t004

containing $200 \mathrm{mM}$ triethanolamine, $\mathrm{pH} 8.25$ for three hours at 298 K. Samples of mNAGS with and without cross-linking reagent were subjected to sodium dodecyl sulfate polyacrylamide get electrophoresis (NuPAGE 4-12\% Bis-Tris gel) in MES SDS buffer (50 mM MES, $50 \mathrm{mM}$ Tris base, 0.1\% SDS, $1 \mathrm{mM}$ EDTA, $\mathrm{pH}$ 7.3) and stained with Coomassie blue. Samples of hNAGS with and without cross-linking reagent were subjected to sodium dodecyl sulfate polyacrylamide get electrophoresis (NuPAGE 4 $12 \%$ Bis-Tris gel) in MES SDS buffer (50 mM MES, $50 \mathrm{mM}$ Tris base, $0.1 \%$ SDS, $1 \mathrm{mM}$ EDTA, pH 7.3) and stained with silver. Size marker controls consisted of proteins with defined molecular weights of protein standards purchased from Invitrogen.

\section{Gel-filtration Chromatography}

Molecular weight of mNAGS and hNAGS were determined with a Superdex 200 HR 10/30 column (Amersham Biosciences) as previously described [5]. The running buffer contains $100 \mathrm{mM}$ $\mathrm{NaH}_{2} \mathrm{PO}_{4} \mathrm{pH} 7.4,150 \mathrm{mM} \mathrm{NaCl}, 10 \%$ glycerol, $1 \mathrm{mM} \beta-$ mercaptoethanol. Thyroglobulin $(669 \mathrm{kDa})$, ferritin $(440 \mathrm{kDa})$, ngNAGS (296.7 kDa), mmNAGS (200.5 kDa) and aldolase (158 $\mathrm{kDa}$ ) were used as protein standards.

Molecular weights of hNAT and the NAT domain of mouse NAGS (mNAT) were determined similarly, but with different protein standards of ovalbumin $(43 \mathrm{kDa})$, albumin $(67 \mathrm{kDa})$, chymotrypsinogen A $(25 \mathrm{kDa})$ and ribonucleause $(13.7 \mathrm{kDa})$.

\section{Crystallization}

Crystals were grown by the sitting-drop, vapor-diffusion method. Before crystallization, the purified protein $(\sim 20 \mathrm{mg} / \mathrm{ml})$ was treated with thrombin (50 units) overnight at $277 \mathrm{~K}$ to remove the his-tag, then incubated with $10 \mathrm{mM} \mathrm{CoA}$, and $20 \mathrm{mM}$ NAG for $30 \mathrm{~min}$. Screening of crystallization conditions was performed using sitting-drop vapor diffusion in 96-well plates (Hampton Research) at $291 \mathrm{~K}$ by mixing $2 \mu \mathrm{l}$ of the protein solution with

\section{References}

1. Slocum RD (2005) Genes, enzymes and regulation of arginine biosynthesis in plants. Plant Physiol Biochem 43: 729-745.

2. Cunin R, Glansdorff N, Pierard A, Stalon V (1986) Biosynthesis and metabolism of arginine in bacteria. Microbiol Rev 50: 314-352.

3. Haskins N, Panglao M, Qu Q Majumdar H, Cabrera-Luque J, et al. (2008) Inversion of allosteric effect of arginine on $\mathrm{N}$-acetylglutamate synthase, a molecular marker for evolution of tetrapods. BMC Biochem 9: 24

4. Qu Q Morizono H, Shi D, Tuchman M, Caldovic L (2007) A novel bifunctional $\mathrm{N}$-acetylglutamate synthase-kinase from Xanthomonas campestris that is closely related to mammalian $\mathrm{N}$-acetylglutamate synthase. BMC Biochem 8. 4 .

5. Shi D, Sagar V, Jin Z, Yu X, Caldovic L, et al. (2008) The crystal structure of Nacetyl-L-glutamate synthase from Neisseria gonorrhoeae provides insights into mechanisms of catalysis and regulation. J Biol Chem 283: 7176-7184.

6. Ramon-Maiques S, Fernandez-Murga ML, Gil-Ortiz F, Vagin A, Fita I, et al. (2006) Structural bases of feed-back control of arginine biosynthesis, revealed by
$2 \mu \mathrm{l}$ of the reagent solution from the sparse matrix Crystal Screens 1 and 2, and Index Screen (Hampton Research). The best crystals were grown from a reservoir solution containing $100 \mathrm{mM}$ Bis-tris, pH 6.5, 35\% PEG3350. Crystals were stick-shaped and took 2-3 days to reach a maximal length of $0.6 \mathrm{~mm}$.

\section{Data Collection and Structure Determination}

Crystals were transferred from the crystallization plate to a well solution supplemented with $25 \%$ glycerol and then frozen directly by liquid nitrogen. Diffraction data were collected at beamline 22ID equipped with MAR300 CCD at the Advanced Photon source (APS), Argonne National Laboratory, USA. All data were processed using the HKL2000 package [25]; statistics are summarized in Table 1. The structure was solved by molecular replacement using Phaser [26,27] based on the NAT domain of mmNAGS/K structure of subunit $\mathrm{X}$ as a search model. After several cycles of refinements with Phenix [28] and model adjustments with Coot [29], NAG was visible in the electron density map and was built into the model. In the last run of the refinement, the translation/liberation/screw parameters were included and refined [30]. Two groups per subunit were selected according to the $\mathcal{N}$-terminal arm (residues 375-469) and the $C$ terminal arm (470-527). Final $R$ and $R_{\text {free }}$ values were $18.4 \%$ and $24.4 \%$, respectively. Refinement statistics for the final refined model are given in Table 1 . The final refined coordinates for NAG bound hNAT and its structure factors have been deposited in RCSB Protein Data Bank with accession code 4K30 and provided as Supplemental Materials.

\section{Supporting Information}

File S1 Coordinate file for the described structure. (PDB)

File S2 Structure factors for the described structure. (CIF)

\section{Acknowledgments}

We thank Dr. David Davies for facilitating the use of the diffraction equipment in the Molecular Structure Section of the National Institutes of Health and Dr. Fred Dyda for help in data collection.

\section{Author Contributions}

Conceived and designed the experiments: DS MT GZ. Performed the experiments: DS GZ ZJ. Analyzed the data: DS GZ ZJ. Contributed reagents/materials/analysis tools: DS GZ. Wrote the paper: DS MT NMA GZ.

the structures of two hexameric $\mathrm{N}$-acetylglutamate kinases, from Thermotoga maritima and Pseudomonas aeruginosa. J Mol Biol 356: 695-713.

7. Min L, Jin Z, Caldovic L, Morizono H, Allewell NM, et al. (2009) Mechanism of allosteric inhibition of N-acetyl-L-glutamate synthase by L-arginine. J Biol Chem 284: 4873-4880.

8. Shi D, Li Y, Cabrera-Luque J, Jin Z, Yu X, et al. (2011) A Novel Nacetylglutamate synthase architecture revealed by the crystal structure of the bifunctional enzyme from Maricaulis maris. PLoS One 6: e28825.

9. Caldovic L, Lopez GY, Haskins N, Panglao M, Shi D, et al. (2006) Biochemical properties of recombinant human and mouse $\mathrm{N}$-acetylglutamate synthase. Mol Genet Metab 87: 226-232.

10. Krissinel E, Henrick K (2007) Inference of macromolecular assemblies from crystalline state. J Mol Biol 372: 774-797.

11. Neuwald AF, Landsman D (1997) GCN5-related histone N-acetyltransferases belong to a diverse superfamily that includes the yeast SPT10 protein. Trends Biochem Sci 22: 154-155. 
12. Dyda F, Klein DC, Hickman AB (2000) GCN5-related N-acetyltransferases: a structural overview. Annu Rev Biophys Biomol Struct 29: 81-103.

13. Hickman AB, Namboodiri MA, Klein DC, Dyda F (1999) The structural basis of ordered substrate binding by serotonin N-acetyltransferase: enzyme complex at 1.8 A resolution with a bisubstrate analog. Cell 97: 361-369.

14. He H, Ding Y, Bartlam M, Sun F, Le Y, et al. (2003) Crystal structure of tabtoxin resistance protein complexed with acetyl coenzyme A reveals the mechanism for beta-lactam acetylation. J Mol Biol 325: 1019-1030.

15. Ah Mew N, Payan I, Daikhin Y, Nissim I, Nissim I, et al. (2009) Effects of a single dose of $\mathrm{N}$-carbamylglutamate on the rate of ureagenesis. Mol Genet Metab 98: 325-330.

16. Kaieda S, Matsui C, Mimori-Kiyosue Y, Ikegami T (2010) Structural basis of the recognition of the SAMP motif of adenomatous polyposis coli by the Srchomology 3 domain. Biochemistry 49: 5143-5153.

17. Macias MJ, Hyvonen M, Baraldi E, Schultz J, Sudol M, et al. (1996) Structure of the WW domain of a kinase-associated protein complexed with a proline-rich peptide. Nature 382: 646-649.

18. Prehoda KE, Lee DJ, Lim WA (1999) Structure of the enabled/VASP homology 1 domain-peptide complex: a key component in the spatial control of actin assembly. Cell 97: 471-480.

19. Schlundt A, Sticht J, Piotukh K, Kosslick D, Jahnke N, et al. (2009) Proline-rich sequence recognition: II. Proteomics analysis of Tsg101 ubiquitin-E2-like variant (UEV) interactions. Mol Cell Proteomics 8: 2474-2486.

20. Adzhubei AA, Sternberg MJ, Makarov AA (2013) Polyproline-II Helix in Proteins: Structure and Function. J Mol Biol 425: 2100-2132.
21. Caldovic L, Morizono H, Tuchman M (2007) Mutations and polymorphisms in the human N-acetylglutamate synthase (NAGS) gene. Hum Mutat 28: 754-759.

22. Caldovic L, Morizono H, Gracia Panglao M, Gallegos R, Yu X, et al. (2002) Cloning and expression of the human $\mathrm{N}$-acetylglutamate synthase gene. Biochem Biophys Res Commun 299: 581-586.

23. Caldovic L, Morizono H, Yu X, Thompson M, Shi D, et al. (2002) Identification, cloning and expression of the mouse $\mathrm{N}$-acetylglutamate synthase gene. Biochem J 364: 825-831.

24. Davies GE, Stark GR (1970) Use of dimethyl suberimidate, a cross-linking reagent, in studying the subunit structure of oligomeric proteins. Proc Natl Acad Sci U S A 66: 651-656.

25. Otwinowski Z, Minor W (1997) Processing of X-ray diffraction data collected in oscillation mode. Methods Enzymol 276: 307-326.

26. Read RJ (2001) Pushing the boundaries of molecular replacement with maximum likelihood. Acta Crystallogr D Biol Crystallogr 57: 1373-1382.

27. Storoni LC, McCoy AJ, Read RJ (2004) Likelihood-enhanced fast rotation functions. Acta Crystallogr D Biol Crystallogr 60: 432-438.

28. Adams PD, Afonine PV, Bunkoczi G, Chen VB, Davis IW, et al. (2010) PHENIX: a comprehensive Python-based system for macromolecular structure solution. Acta Crystallogr D Biol Crystallogr 66: 213-221.

29. Emsley P, Cowtan K (2004) Coot: model-building tools for molecular graphics. Acta Crystallogr D Biol Crystallogr 60: 2126-2132.

30. Winn MD, Isupov MN, Murshudov GN (2001) Use of TLS parameters to model anisotropic displacements in macromolecular refinement. Acta Crystallogr D Biol Crystallogr 57: 122-133. 